\title{
«Des vers hors de rime, et de raison » : réflexions poétiques et définition de soi dans Les Amours de Christofle de Beaujeu
}

\author{
UGO PAIS
}

Université Lumière-Lyon 2

Lu par un lectorat restreint composé de critiques et d'amateurs de curiosités littéraires, l'ouvre de Christofle de Beaujeu a souvent fait l'objet d’un jugement négatif. "Torrentueux » ou "médiocre » sont les adjectifs les plus employés à son endroit. Le recueil des Amours, principal texte de Beaujeu, contient quelques textes introductifs où l'auteur tente de définir le lectorat qu'il attend en mettant en avant ce qu'il considère comme ses atouts tout en essayant de justifier ce qu'il perçoit lui-même comme des obstacles à la compréhension. Le but de cet article est de se demander quels sont ces obstacles et comment la vision que le poète a de son art entre très vite en conflit avec le nouveau goût qui émerge à la fin du XVI siècle.

Read by a limited readership of critics and connoiseurs of literary curiosities, Christofle de Beaujeu's work has often been subject to negative judgment. "Unrestrained" or "mediocre" are the adjectives most often applied to it. Beaujeu's major work, the collected Amours, contains a few introductory texts where the author attempts to define his expected readership by pointing out what he considers to be his virtues, while at the same time trying to justify what he himself feels will be hindrances to their understanding. The aim of this article is to inquire into the nature of these hindrances and how the poet's vision of his art very quickly comes into conflict with the new aesthetic values emerging at the end of the sixteenth century.

L e cas de Christofle de Beaujeu appartient à la longue liste des auteurs passés écho lors de sa publication et en a eu très peu par la suite. Elle pose le problème de l'existence même d'un lectorat puisqu'on ne peut que supposer celui-ci à travers les lettres préfacielles et la réédition du sonnet A Madame, à qui je fis fort bien un enfant dans le recueil collectif publié en 1615 par Antoine Estoc nommé Les satyres bastardes et autres cuvres folastres du Cadet Angoulevent ${ }^{1}$. L'essentiel des lecteurs de Beaujeu depuis le XVII ${ }^{\text {e }}$ siècle appartient donc au

1. Frédéric Lachèvre, Les recueils collectifs de poésies libres et satiriques publiés depuis 1600 jusqu'à la mort de Théophile (1626) (Genève : Slatkine Reprints, 1968), 93. Les satyres bastardes et autres œuvres folastres du Cadet Angoulevent (Paris : Antoine Estoc, 1615) : https://gallica.bnf.fr/ark:/12148/bpt6k72621h. imagerenaissance 
cercle restreint des bibliophiles, des biographes ou des critiques littéraires, depuis l'Abbé Gouget jusqu'à Gisèle Mathieu-Castellani² en passant par les auteurs de dictionnaires biographiques des $\mathrm{XIX}^{\mathrm{e}}$ et $\mathrm{XX}^{\mathrm{e}}$ siècles${ }^{3}$, Viollet-le-Duc, Roubaud et Jean-Paul Barbier ${ }^{4}$.

De plus, si depuis l'anthologie d'Albert-Marie Schmidt, L'Amour noir ${ }^{5}$, la critique littéraire moderne tente de mettre en exergue les qualités de la poésie de Beaujeu, celle-ci a longtemps été jugée exécrable. Qu’il nous suffise de citer, par exemple, ce mot trouvé, à propos des Amours, dans un recueil de biographies publié en 1811 : «On y trouve des odes, des sonnets, des élégies. Toutes ces pièces sont au-dessous du médiocre $»^{6}$. L'œuvre de Beaujeu est donc restée jusqu'à aujourd'hui un objet manié par une petite partie de la critique ou par les amateurs de curiosités littéraires.

Cet état des lieux ne doit pas faire oublier que Beaujeu, confiant dans la bienveillance du lectorat, s'interroge sur la réception de son œuvre et tente d'orienter le lecteur à l'orée d'un recueil qu'il juge lui-même quelque

2. Voir Abbé Goujet, Bibliothèque françoise, 18 vols. (Paris : H.-L. Guérin et P.-G. Le Mercier, 1752), 13 : 297-303. Giselle Mathieu-Castellani, quant à elle, s'est penchée sur l'œuvre de Beaujeu dans Les Thèmes amoureux dans la poésie française (1570-1600) (Paris : Klincksieck, 1975) et, plus récemment, Le Rossignol poète dans l'Antiquité et à la Renaissance (Paris : Classiques Garnier, 2016) ; elle a également édité une sélection de ses poèmes dans Eros Baroque. Anthologie thématique de la poésie baroque (Paris : 10/18, 1979) et Entouré de silence (Paris : La Différence, 1995).

3. Nouvelle Biographie universelle depuis les temps reculés jusqu'à nos jours, avec les renseignements bibliographiques et l'indication des sources à consulter, éd. M. le Dr Hoeffer, 9 vols. (Paris : Firmin Didot Frères, 1852), 3 : 930 ; Dictionnaire de biographie française, éd. MM. Prevost et Roman D’Amat, 21 vols. (Paris : Librairie Letouzet et Ané, 1951), 5 : 1098 ; Grand dictionnaire universel du XIXe siècle, éd. Pierre Larousse, 16 vols. (Genève : Slatkine, 1982 [1 $1^{\text {ère }}$ éd. Administration du Grand Dictionnaire Universel, 1867]), $5: 436$.

4. Emmanuel Louis Nicolas Viollet-le-Duc, Catalogue des livres composant la bibliothèque poétique de M. Viollet-le-Duc (Paris : Hachette, 1843), 299 ; Jacques Roubaud, Soleil du soleil. Le sonnet français de Marot à Malherbe. Une anthologie (Paris : P.O.L., 1990), 225-232 ; Jean-Paul Barbier, Ma Bibliothèque poétique, successeurs et contemporains de Ronsard (Genève : Droz, 2001).

5. Albert-Marie Schmidt, L’Amour noir. Poèmes baroques, prés. J. Roubaud (Paris : Slatkine, 1982 [1 ${ }^{\text {ère }}$ éd. 1959]), 46 et 49.

6. Biographie universelle ancienne et moderne, 52 vols. (Paris : Michaud Frères, 1811), 3 : 630. À propos du premier chant de l'épopée nommée La Suisse, l'auteur de cette notice ajoute : « Les suivants n'ont jamais paru, et on ne doit pas en avoir de regret ». Ce n'est ici qu'un exemple des nombreux jugements négatifs qu'on trouve par exemple chez Goujet, Viollet-le-Duc ou Roubaud. 
peu complexe. De fait, dans le "Au Lecteur » général du recueil ${ }^{7}$, certaines personnes bien définies sont considérées par le locuteur comme capables de comprendre la poésie beaujelienne. Ses œuvres ont été écrites dans un cadre intime qui exclut a priori quiconque n’a pas fait partie du cercle des « Déesses du temple $»^{8}$. L'auteur caractérise ainsi les dames qu'il a honorées d’un culte particulier9. La lecture de ses œuvres est accessible à elles seules. Bien conscient de ce problème, Beaujeu nous indique qu'il a essayé de changer cet état de fait, mais qu'il a eu peur de tout "gaster » et qu'il a préféré s'abstenir de corriger sa poésie. Il distingue, dès lors, trois catégories de lecteurs et estime leurs capacités respectives à accéder à son œuvre :

Toutesfois j'espere que les sçavans hommes me tiendront pour desireux de sçavoir, les mediocres pour compagnon, et les ignorans pour elegant qui est la plus grande partie du monde, et qui durera le plus, si les armes ont tousjours autant de credit en France, qu'elles ont eu depuis que je me suis mis à escrire, ayant laissé maintesfois ancre, livre et papier pour courir aux alarmes, que je voudrois estre aussi esloignées de nous, qu'elles en sont pres $[\ldots] .{ }^{10}$

Cette typologie sommaire se termine sur une hyperbate qui donne à la fois une caractérisation de la plupart des lecteurs et une définition de ce qu'est l'auteur et du type de poète qu'il est. Bien plus, il fait ici une sorte de tableau de la société française de l'époque, partagée, selon lui, entre des savants qui ne sont pas militaires et des militaires ignorants. Seul un petit nombre de ceux qui s'adonnent au métier des armes semble trouver grâce à ses yeux et fait l'objet de son attention. Beaujeu réactive ici une ligne de partage sensible à la Renaissance et surtout dans la seconde moitié du siècle, celle qui existe entre la noblesse militaire, héritière des traditions et des valeurs de la chevalerie et la noblesse dite de robe ou, à tout le moins, celle qui voit l'éducation humaniste

7. Christofle de Beaujeu, Les Amours (Paris : Didier Millot, 1589), f. 6 r.

8. Beaujeu, Les Amours, f. 6 ro.

9. Voir Beaujeu, f. $4 \mathrm{r}^{\mathrm{o}}, 32 \mathrm{v}^{\mathrm{o}}, 61 \mathrm{v}^{\mathrm{o}}$ et $62 \mathrm{v}^{\mathrm{o}}$. On trouve le vers : " O beaux yeux que je sers, comme Dieux en un Temple » qui explicite l'expression. De plus, contrairement à beaucoup de chansonniers renaissants, Les Amours de Christophe de Beaujeu ne sont pas consacrés à une seule amante. Le nom de « Rose » est le plus fréquent mais on trouve aussi ceux de « Moléon » ou « Du Tertre ».

10. Beaujeu, Les Amours, f. 6 ro. 
en plein essor et la pratique des arts comme indispensable à la construction de l'ethos du noble ${ }^{11}$. Chassée de certains de leurs postes traditionnels par des érudits roturiers, une frange de la noblesse se réfugie dans l'exaltation de ses valeurs guerrières ${ }^{12}$ et vit les guerres de Religion comme une chance d'égaler les exploits militaires de ses ancêtres pendant les guerres d'Italie ${ }^{13}$. Beaujeu se voit comme le représentant d'une minorité, et celle-ci, où il trouverait son lectorat privilégié, se réduit en même temps qu'elle devient une entité instable et difficile à définir. Apparaît ici un conflit vraisemblablement vécu par le poète lui-même, par lequel il tente d'organiser la réception de l'œuvre. Cependant, ce conflit est aussi vecteur de partis pris littéraires puisque Beaujeu semble tenter de concilier son propre état de militaire et son activité poétique en opérant, on va le voir, des choix stylistiques lui permettant de revendiquer le premier à travers la seconde. Cette opération délicate oblige le poète à mettre en exergue certains de ces choix qui, s'ils sont cohérents avec les revendications esthétiques de l'auteur, ne nous paraissent pas tout à fait conforme à l'évolution du goût du temps. C'est tout d'abord à travers l'emploi du terme « torrent » et ses connotations que nous aborderons cette position complexe avant de nous pencher sur la reprise de l'opposition, traditionnelle dans le discours critique de la fin du XVI e siècle ${ }^{14}$, entre style " rude » et style " doux ». Dans les deux cas, nous tenterons de mettre en parallèle ce discours beaujelien avec ce que dit Ronsard, alors érigé en véritable modèle poétique ${ }^{15}$, de ses propres œuvres dans les dernières années précédant sa mort en 1585, pour mieux apprécier l'originalité de la posture de notre auteur.

11. Arlette Jouanna, "La noblesse française et les valeurs guerrières au XVI siècle ", in L'Homme de guerre au XVI siècle, actes du colloque de l'Association RHR, Cannes, 1989, éd. Gabriel-André Pérouse, André Thierry et André Tournon (Saint-Étienne : Publications de Saint-Etienne, 1992), 214-215.

12. Jouanna, «La noblesse française... », 216.

13. Jouanna, « La noblesse française... », 208-209.

14. Voir sur ce point Claude Faisant, Mort et résurrection de la Pléiade, publié par Josiane Rieu avec la collaboration de James Dauphiné, Guy Demerson, Claude Digeon et al. (Paris : Honoré Champion, 1998), 66.

15. Sur la place de Ronsard dans la poésie des vingt dernières années du XVI ${ }^{\mathrm{e}}$ siècle, voir Faisant, Mort et résurrection de la Pléiade, 51-53. 


\section{L'image du « torrent $»^{16}$}

Christofle de Beaujeu prend le parti de nous présenter de manière peu engageante ses écrits et insiste sur les problèmes de compréhension auxquels le lecteur va se heurter. L'expression la plus explicite à ce sujet est celle que l'on trouve dans notre titre, où Beaujeu annonce que le lecteur pourra trouver des « vers hors de rime, et de raison $»^{17}$. Elle renvoie à un type de discours « déraisonnable voire absurde », comme le propose le $\mathrm{DMF}^{18}$. Le nom « rime» est cependant complexe à définir à la Renaissance et entretient, surtout en contexte poétique, des liens problématiques avec la " raison $»^{19}$. En l'occurrence, il s'agit ici d'un appel à la bienveillance du lecteur face à un texte où la forme tout autant que le fond peuvent être défectueux. Beaujeu explique ces «vers hors de rime, et de raison » par la présence régulière « des noms tournez, Anagrammes, Significations, ou jargon » qui, selon lui, « ne sont point intelligibles, qu'aux Deesses du Temple où mes sacrifices estoient faits $»^{20}$. La compréhension littérale du texte peut être troublée par un jeu trop poussé sur le signifiant, auquel l'auteur aurait sacrifié la clarté du texte. Le vers n'est qu'un prétexte à la dissimulation d'un nom de famille ou d'un mot, créant ainsi un effet de connivence avec celles et ceux qui sont capables de le déchiffrer. L'exigence de communication, essentielle à nos yeux de critiques imprégnés des conceptions classiques ${ }^{21}$, ne semble pas celle de Beaujeu, bien qu'il commence à y être sensible. Pour lui, c'est le plaisir

16. Le mot est utilisé dans bien des contextes et en particulier pour évoquer la violence de l'expression dans la littérature pamphlétaire. Nous ne nous intéresserons ici qu'à ses emplois dans la qualification de la poésie lyrique et, plus spécifiquement, dans celle de la poésie amoureuse.

17. Beaujeu, Les Amours, f. 6 ro. L'on ne saurait, de fait, prendre cet avertissement trop au sérieux au vu du nombre de vers faux ou qui riment de façon très approximative, que l'on trouve un peu partout dans le recueil.

18. Dictionnaire du Moyen Français consulté en ligne le 21/11/2017 à l'adresse http://www.atilf.fr/dmf.

19. Sur cette épineuse question, voir, pour la période qui précède le texte de Beaujeu, l'article de Guillaume Berton " "Je rime en prose (et peult estre en raison)" : Marot et la rime ", Bibliothèque d'Humanisme et Renaissance 70.1 (2008) : 7-32 ; et, pour celle qui le suit, celui de Delphine Reguig "Froideur et saveur de la rime chez Boileau ", in L'Épithète, la rime et la raison. La lexicographie poétique en Europe, XVI $-X V I I^{e}$ siècles, éd. Sophie Hache et Anne-Pascale Pouey-Mounou (Paris : Classiques Garnier, 2015), 367-382.

20. Beaujeu, Les Amours, f. 6 ro.

21. C'est ainsi que nous pouvons éclairer les jugements négatifs fortement répandus sur l'œuvre de Beaujeu, qu'il nous est bien difficile d'appréhender par-dessus les théories classiques. 
du codage du texte et du secret qui prime. Une attention soutenue est donc demandée au lecteur, ainsi qu'une bienveillance de tous les instants car ce qu'il ne comprend pas pourrait bien dissimuler un sens caché. Cet appel aux qualités exégétiques du lecteur, adossé à la revendication de la nouveauté de l'œuvre, peut être rapproché de l'ode "A sa muse » de Ronsard, dans laquelle le poète vendômois évoque violemment ses volontés en tant que poète et son rapport à ses pairs présents ou passés :

Ne suy ny le sens, ny la rime,

Ny l'art du moderne ignorant,

Bien que le vulgaire l'estime,

Et en béant l'aille adorant. ${ }^{22}$

En 1550, Ronsard revendiquait son désintérêt envers une poésie de la clarté et de la régularité technique au détriment de la puissante imagination du poète. Taillée pour le combat, cette strophe au premier vers programmatique mettait déjà à distance la catégorie des "ignorants ", dans une volonté de s'écarter de la poésie à la mode pour créer une œuvre à la fois originale et éloignée des canons. Si Beaujeu fait des «ignorants » les militaires qui refusent la culture humaniste, là où Ronsard cherche à affirmer la nouveauté du jeune groupe de poète qu'il appellera bientôt " Pléiade ", tous deux mettent à l'écart cet « art » de la poésie qui leur semble entraver leurs capacités d'expression.

Christofle de Beaujeu se livre dès lors à une mise en scène de la production de son œuvre qui apparaît en premier lieu dans le titre de l'ensemble de cent vingt-et-un sonnets, nommé Torrent des Sonets, occupant la première partie du recueil. Il est évident qu'à l'instar de l'Hécatombe à Diane d'Agrippa d'Aubignéz ${ }^{23}$ le titre Torrent des Sonets est déjà en lui-même programmatique et donne à cette partie des Amours un caractère d'emblée impétueux et bouillonnant. Avant de nous intéresser aux différentes métaphores que Beaujeu développe à partir du terme « torrent » pour expliquer la création de son œuvre,

22. Pierre de Ronsard, Euvres complètes, vol. 1, éd. Paul Laumonier (Paris : Société des Textes Français Modernes, 1973), 236.

23. Ce recueil de cent sonnets met largement en scène le sacrifice du locuteur sur l'autel de son amour pour la dame, vérifiant ainsi le terme d'« hécatombe » employé dans le titre. Voir en particulier sonnets XCIV, XCVI et XCVII dans Agrippa d'Aubigné, L'Hécatombe à Diane, éd. Julien Goeury (Saint-Étienne : Presses de l'Université de Saint-Étienne, 2007), 191, 194 et 196. 
il convient de donner un bref aperçu d'un substantif qui est, en 1589, tout sauf neutre : comme le rappelle Perrine Galand-Hallyn, depuis l'Antiquité, ce terme fait partie d'une métaphore plus vaste qui vise à rapprocher les styles poétiques « et, plus généralement, toute démarche intellectuelle, [...] [de] la métaphore du cours d'eau, au flux calme ou rapide, aride ou abondant $»^{24}$. De fait, le terme faisant écho au sonnet liminaire de la première édition des Quatre premiers livres des Odes de Pierre de Ronsard, Vendomois écrit par Du Bellay, Beaujeu se montre ici en lecteur de ses aînés, héritier des poètes de la Pléiade et engageant un véritable dialogue avec les plus illustres d'entre eux. Rien de bien original jusque-là. On trouve explicitement le terme «torrent », qui plus est au premier vers du sonnet de Du Bellay :
Comme un torrent qui s'enfle et renouvelle $[\ldots]$
Tes vers Ronsard, qui par source immortelle
Du double mont sont en France venus
Courent (hardis) par sentiers inconnus
De méme audace, et de carrière telle. ${ }^{25}$

L'image fluviale est essentiellement développée pour faire de Ronsard le digne héritier de Pindare et le nouvel élève des Muses. C'est tout le processus d'imitation et d'inspiration défendu par la Pléiade qui prend corps dans cette crue fantastique, menant des sources consacrées aux muses sur le mont Parnasse à cette nouvelle terre poétique qu'est la France. Ce flot ronsardien se veut impétueux, «hardi », " audac[ieux] » et même «inconnu », c'est-à-dire sans précédent. Malgré le rappel des origines de la verve du nouveau poète, celuici apparaît bien comme un artiste à l'originalité certaine, certes héritier mais surtout inventeur d'une manière nouvelle qui n'entend pas se dissimuler. Cette prise de position est conforme à celle de la Défense et illustration de la langue française qui mettait en avant la nécessité de la lecture des Anciens, tout en affirmant la modernité des jeunes poètes. Cependant, malgré son ton laudatif, ce sonnet de Du Bellay n'aura pas la fortune des odes qu'il introduit, puisqu'il est absent de la seconde édition des Odes en 1553. S’il est réimprimé lors de la 
troisième édition de cet ouvrage, en $1555^{26}$, et dans les éditions de 1560 et 1567 des Euvres $^{27}$, il n'est plus réimprimé par la suite dans les rééditions données du vivant de Ronsard. Le sonnet n'est pas non plus présent dans les éditions posthumes des Euvres de 1587 ou $1607^{28}$. Il faudra, en fait, attendre l'édition de Prosper Blanchemain, en $1866^{29}$, pour qu'il réapparaisse ${ }^{30}$. Le texte de Du Bellay n'est pas le seul à subir un rapide ostracisme.

À la fin du recueil, Ronsard lui-même n'hésite pas à reprendre l'image qui organise le sonnet liminaire dans son ode «A sa muse » déjà citée, lorsqu'il s'écrie :

Grossi-toy, ma Muse Françoise,

Et enfante en vers resonant,

Qui brusle d'une telle noise

Qu'un fleuve debordé tonant

Alors qu'il saccage et emmeine,

Pillant de son flot, sans merci,

Le thresor de la riche pleine,

Le bœuf et le bouvier aussi. ${ }^{31}$

Si l'hyperonyme «torrent » n'apparaît pas ici, le locuteur déroule sous les yeux du lecteur une scène de crue qui n'oublie ni la violence de ce type d'événement ni son aspect sonore. Ronsard insiste sur le bruit, voire sur le choc auditif que, tel le torrent, ses vers produisent, « resonant», « tonant » d'une « noise » bien particulière. Bien plus, cette ode accentue encore les traits les plus marquants de l'image développée par Du Bellay : c'est un véritable raz-de-marée que souhaite ici Ronsard, annihilant la tranquillité du simple travailleur et détruisant sa

26. P. de Ronsard, Les quatre premiers livres des odes de P. de Ronsard (Paris : chez la Veuve M. De La Porte, 1555).

27. P. de Ronsard, Euvres complètes (Paris : G. Buon, 1560), t. II, NP (9) et P. de Ronsard, Euvres complètes (Paris : G. Buon, 1567), t. I, $5 \mathrm{r}^{\circ}$.

28. P. de Ronsard, Euvres complètes (Paris : G. Buon, 1587) et P. de Ronsard, CEuvres complètes (Paris : N. Buon, 1609).

29. P. de Ronsard, Euvres complètes, nouvelle édition, publiée sur les textes les plus anciens avec les variantes et des notes par M. Prosper Blanchemain (Paris : P. Jannet, 1857-1867), t. I (1857), p. xxvi.

30. Ronsard, Euvres complètes (Paris : Société des Textes Français Modernes), vol. 1, 56.

31. Ronsard, Euvres complètes, vol. 2, 236. 
production. Si le pillage du "thrésor " renvoie à l'imitation des Anciens, le locuteur furieux se veut plutôt à la source d'une table rase qui permette de nouvelles fondations. La poésie ainsi présentée est une force d'entraînement, à l'avant-garde de la modernité artistique à la fois enrichie des productions passées et destructrice de celles-ci. Le mot « torrent » et son utilisation en tant que métaphore de la production poétique fait donc partie de l'imaginaire de la Pléiade, mais il est très vite rejeté par Ronsard. Cette ode subira un sort encore moins enviable que le sonnet cité précédemment puisqu’elle est supprimée dès l'édition de l'ouvrage en 1553. Elle est cependant rééditée en 1592 dans l'édition lyonnaise ${ }^{32}$ des CEuvres, avant de se trouver dans le Recueil des pièces retranchées des éditions de 1609 et 1623 . Si ce parcours dans les modifications apportées par Ronsard sur ses œuvres dès les premières rééditions nous permet de faire valoir la finesse et la cohérence de son travail de correction, on peut aussi y déceler les pratiques de lecteur des poètes qui suivent la Pléiade. De fait, si la vision du Vendômois sur ses propres œuvres paraît s'être modifiée $e^{33}$, en revanche les choix lexicaux de Beaujeu nous semblent révéler qu'il est bien loin de partager les repentirs du Prince des poètes : pour lui, la création originale se dit toujours à travers l'image du torrent ${ }^{34}$. Ce fait nous semble d'autant plus intéressant que, dans les années 1580, Ronsard revient, de manière détournée, sur l'emploi du terme « torrent » en contexte poétique. Dans sa Vie de Ronsard de 1586, Claude Binet mentionne quelques phrases du maitre où le mot réapparaît pour tancer vertement les poètes récents :

Ils ont, me disoit-il, l'esprit plus turbulent que rassis, plus violent qu'aigu, lequel imite les torrents d'hyver, qui attrainent des montaignes

32. P. de Ronsard, Euvres complètes (Lyon : T. Soubron, 1592).

33. Les connotations attachées par Ronsard au terme « torrent » dans ses emplois méta-poétiques, sont, dès les années 1550, ambiguës. Il évoque ainsi, dans «L'Élégie à Chretophle de Choiseul », « les repliz recourbez / Des torrens de Pindare en profond embourbez, / Obscurs, rudes, facheux, et ses chansons congnues [...] », qui l'intéressent moins que le " doux Anacreon ». On retrouve ici le lien entre le style de Pindare et la métaphore du torrent qui est déjà développée par Quintilien (Institution oratoire, X, 1 et XI, 3), Horace (Odes, IV, 2, v. 1-8) et Ronsard (Euvres complètes, vol. 8, 351-358, v. 80-81).

34. De ce point de vue, Beaujeu n'est guère original. De fait, Claude Faisant, Mort et résurrection de la Pléiade, 41-42, insiste sur l'amour des humanistes des années 1580 pour le Ronsard des Odes et des Hymnes, dont les premières éditions datent de 1550 et 1552, ainsi que leur surprise face aux corrections effectuées par le poète sur ses propres œuvres. La nostalgie est d'autant plus forte que les critiques du temps ne voient personne pour reprendre le flambeau. 
autant de boüe que de claire eauë : voulant éviter le langage commun, ils s'embarassent de mots et de manières de parler dures, fantastiques, et insolentes $[\ldots] .^{35}$

Si Ronsard fait sans doute ici allusion aux poètes qui circulent dans l'entourage de Desportes, à la cour d'Henri III, la métaphore employée nous intéresse, car, à quelque quarante ans de distance, ce qui était positif est devenu négatif pour le poète vieillissant ${ }^{36}$. De manière significative, le complément du nom « d'hyver» qui accompagne le substantif «torrent » introduit dès l'abord l'idée d'un déclin, voire d'une stérilité. Cette idée est aussitôt renforcée par une subordonnée relative, et celle-ci remplace la créativité syncrétique que symbolise la crue de l'«Ode à sa muse » par une violence trouble; les raisons de cette violence n'ont rien à voir avec une fureur inspirée, elle s'explique par les lubies d'un poète désormais à moitié fou. C'est bien le sens de la triade d'adjectifs finale, qui superpose à la complication du style l'inconvenance de celui-ci.

Il est bien sûr impossible d'affirmer que Beaujeu ait lu ces pages de la Vie de Pierre de Ronsard de Claude Binet, mais il n'en reste pas moins que la métaphore du "torrent » paraît avoir désormais basculé du côté des défauts poétiques à éviter. Et pourtant, Beaujeu insiste fortement sur ce terme, qui semble le seul à même de décrire les caractéristiques de son esthétique.

Ainsi, dans le "Au Lecteur » général des Amours, le poète inclut le mot «torrent» dans une métaphore qui sert de récit de la production du texte. Dans l'image ainsi filée, il parle des « flots de [son] amour » qui sont « roulez » ${ }^{37}$ d'une manière bien particulière avant de constater :

35. Claude Binet, La Vie de Pierre de Ronsard, éd. Paul Laumonier (Genève : Slatkine, 1969 [1909]), 39. La critique moderne a pu considérer Beaujeu dans les mêmes termes que ceux employés par Ronsard. Ainsi, Jacques Roubaud s'exclame à propos du Torrent des Sonets dans son Soleil du soleil, 226 : «Que d'eaux bourbeuses il entraîne avec soi ! ». Bien qu'il soit probable qu'il connaisse le passage rapporté par Binet, on ne peut que remarquer l'homogénéité des connotations liées au terme « torrent ", à travers presque 450 ans d'histoire de la langue.

36. Pour le dire plus précisément, c'est sans doute la poésie amoureuse pratiquée par la nouvelle génération - celle qui succède à Ronsard - qui est ici qualifiée de torrentueuse. Si le terme peut correspondre aux odes en 1550, en revanche, dans la bouche de Ronsard, il qualifie négativement la poésie néo-pétrarquiste trente ans plus tard. En le reprenant à son compte, Beaujeu semble tenter de faire une synthèse entre l'évolution du style pétrarquiste en 1580 et les revendications esthétiques des jeunes poètes de 1550 .

37. Beaujeu, Les Amours, f. 6 ro. 
L'on peut juger bien aisément aussi à lire les diversitez de mon amour, de quelle source sont sortis mes vers, pour estre tres-rudes et remplis d'une legitime consonance, suffisante preuve qu'ils ont ruisselé au long d'un rivage bordé de cailloux ennemis, qui les ont attendus et fait passer entre eux pour leur faire desplaisir, en mon exil qui a esté aussi long et facheux que le pays où j'ay esté relegué est rude. ${ }^{38}$

Si la métaphore de la crue est ici exclue par le poète, c'est en revanche le rapport du torrent à l'originalité du style et à son caractère pragmatique - le lecteur n'y peut rester insensible - qui est largement mis en avant. L'adjectif « rude » qui termine la phrase et reprend l'intensif «tres-rude » qui débutait la description des vers rappelle le terme « dure » qu'on trouvait dans les paroles de Ronsard rapportées par Binet. Là où Du Bellay voulait placer son rival au sommet des poètes français en digne héritier des grands poètes de l'Antiquité, Beaujeu semble tenter de faire une synthèse entre les deux poètes et faire ainsi valoir la sincérité de son expression. Il développe la métaphore en employant l'isotopie du fleuve de montagne : " source », " ruisselé », " rivage », "bordé », " cailloux ", tous ces termes permettant une mise en scène de la souffrance dans le texte à travers les " cailloux ennemis » sur lesquels se heurtent les vers du poète et qui leur donnent un aspect peu engageant. On peut également noter que si Beaujeu tente de réactiver une image passée de mode en 1589, il entre aussi en conflit avec les attentes poétiques de ses contemporains, poètes et lecteurs. De fait, outre le terme " torrent », c'est le mot «fantastique » qui devient, lui-aussi, le propre du poète. Une rapide recherche sémantique dans le recueil révèle sa présence par huit fois, dont six pour qualifier directement le locuteur. Apparaissant également six fois à la rime, le terme vient caractériser un état mental proche de la folie ${ }^{39}$. Le locuteur s'exclame ainsi : « Je faignois n'aimer point, mais mon mal fantastique / Monstroit bien qu'au cerveau estoit ma sciatique ${ }^{40}$. Par rapport aux remarques de Ronsard, c'est ici le sujet entier qui est contaminé par un « mal » qui touche physiquement le locuteur et surtout

38. Beaujeu, Les Amours (Paris: Société des Textes Français Modernes), f. 5 ro-f. 5 vo.

39. Par comparaison, une recherche dans la base Frantext montre que le terme n'apparait qu'une seule fois dans Les Amours, telles que publiées par Ronsard en 1553. De plus, on ne le retrouve que trois fois dans l'ensemble des textes du XVI e siècle que contient cette base : ATILF (Analyse et Traitement Informatique de la Langue Française), Base de données Frantext, https://www.frantext.fr/.

40. Beaujeu, Les Amours, f. 65 ro. 
les nerfs, se transmettant à travers tout le corps. L'impossibilité de la feinte, liée au caractère visuel immédiat de la douleur, semble répondre à Ronsard, qui critiquait de telles manières de parler : non seulement ce ne sont pas que procédés de style mais, en plus, ceux-ci sont montrés comme l'expression sincère et inévitable d'un bouleversement intérieur général.

Cette idée est accentuée dans l'ensemble du recueil par l'articulation de l'image du «torrent » avec celle du lieu même de l'écriture, ce lieu de l'exil, pays " rude », la Suisse, où Christofle de Beaujeu a probablement vécu au tournant des années $1580^{41}$, pour des raisons qui restent encore à préciser. Au-delà des parallèles avec Ronsard ou Du Bellay, que son vocabulaire méta-poétique fait naître, Beaujeu ancre la métaphore dans une narration de sa propre expérience. L'adjectif qualifie ce pays dans la première élégie du recueil, qui contient un long discours de Jupiter narrant la vie du locuteur :
Il estoit exilé de son pays François,
Et n'avoit pour seurté, que les monts et les bois,
Privé de tout le sien. Or pour s'oster de peine,
Il laissa sa maison, et traversa la plaine
De la froide Champagne, et tira droit aux monts
De la rude Suisse $[\ldots] .^{42}$

La scène met en parallèle un manque sentimental et spirituel qui atteint le locuteur et un manque physique matérialisé par l'exil et la fuite hors de la nation, voire hors de la civilisation ${ }^{43}$, puisqu'il se trouve dans les " monts et les bois ». Cette référence à une forme de sauvagerie naturelle n'est pas sans évoquer le

41. Cet épisode de l'exil en Suisse intervient à de multiples reprises dans les Amours. Voir par exemple le sonnet $A u$ Roy f. $6 \mathrm{v}^{\circ}$, l'élégie f. $14 \mathrm{r}^{\circ}$, l'élégie f. $31 \mathrm{r}^{\circ}$, l'élégie f. $146 \mathrm{v}^{\circ}$, etc. Quant au caractère rude du pays, Beaujeu nous en livre les raisons dans cette strophe (Beaujeu, Les Amours, f. $30 \mathrm{r}^{\circ}$ ) : « Mes chevaux non lassez de me faire service, / Firent bientost retour au pays de Suisse, / Où sans commoditez qui fait l'homme durer, / Vainqueur de ma misere il fallut endurer / L'Hyver aspre suivant, la froideur de la neige, / Les estrangeres mœurs, qu'une mort seule pleige, [...] ».

42. Beaujeu, Les Amours, f. 13 ro.

43. Cette mise en scène de soi rapproche bien sûr le poète d'Ovide mais aussi, pour mentionner des auteurs plus proches de Beaujeu à la fois chronologiquement et poétiquement, de Pétrarque et Du Bellay. Pour une mise au point générale sur la question, voir George Hugo Tucker, Homo Viator : Itineraries of Exile, Displacement and Writing in Renaissance Europe (Paris : Droz, 2003), en particulier 37-52 et 239-267. 
« rivage bordé de cailloux ennemis $»^{44}$. L'exil devient la matrice de l'expérience poétique du locuteur, la seconde se développant comme la trace du premier. En fait, cette image méta-poétique articule rêverie sur une séquence biographique et revendication esthétique, en prenant pour charnière le terme " rude », qui joue un rôle central car son sémantisme s'applique à divers référents et permet de faire le lien entre eux.

Dès lors, on comprend mieux l'importance du syntagme "legitime consonance ", qui apparaissait dans le "Au Lecteur " précédemment cité. Reprenant à son compte la " noise » des vers de Ronsard, Beaujeu insiste sur la matérialité de ses vers, reconnaissable entre toutes, puisqu'elle est liée à l'« exil [...] long et facheux ». Bien plus, cette signature sonore n'est nullement le fruit du hasard ou d'un artifice poétique, puisqu'elle est à la fois « legitime » et «suffisante preuve » de la peine du poète à produire des vers et des difficultés de sa propre vie. Les vers du Torrent sont donc les témoins des souffrances subies par le locuteur, en ce sens qu'ils nous sont montrés comme consubstantiels à leur auteur : remettre en cause la sincérité de ces vers, c'est remettre en cause l'intégrité du poète lui-même. Beaujeu justifie son écriture par la violence des épreuves qu'il a traversées. Paradoxalement, il s'oppose à la catégorie des «savants » que nous avons décrite plus haut comme opposée à l'activité militaire, puisque celle-ci était matrice de l'ignorance. Il nous semble qu'en revendiquant un lien organique entre le caractère complexe de ses vers et la difficulté d'un exil, Beaujeu se détache de l'évolution d'une certaine poésie érudite qui serait l'apanage des « savants ». Au contraire, malgré ses appels à la bienveillance du public, il voit comme positif le caractère tortueux de sa poésie, qui est le signe de sa sincérité. Il nous semble dès lors chercher à faire sien un public dont le goût naturel n'est pas en accord avec les pratiques poétiques des années $1580^{45}$, la métaphore du «torrent » servant en quelque sorte à séduire le lecteur humaniste héritier des revendications poétiques des années 1550. Aussi cette position conduit-elle Beaujeu à se positionner contre le style doux en faveur à l'époque, pour tenter de mettre en avant l'aspect rugueux de son écriture.

44. Voir note 38 .

45. Celui-ci, comme le rappelle bien Claude Faisant, Mort et résurrection de la Pléiade, 66, est loin de faire l'unanimité en ces dernières années du XVI $\mathrm{XI}^{\mathrm{e}}$ siècle où les lecteurs sont à la fois d'" honnêtes gens, [...] [des] doctes et [de] purs mondains », les avis fluctuant selon les lectures et les moments. 


\title{
Le rude et le doux
}

On peut tout d'abord rappeler que les premiers lecteurs auxquels s'adresse le locuteur sont « les Deesses du Temple où [s] es sacrifices estoient faits ${ }^{46}$. On a bien là la proclamation d'une irréductible originalité, qui a trait à la profonde singularité de l'œuvre, adossée à la singularité des épreuves vécues. Au demeurant, le mot "sacrifices " révèle tout son sens. Ces " sacrifices ", poèmes consacrés aux amantes du locuteur, sont les martyrs - au vrai sens de " témoins » donnant leur vie pour prouver leur foi - qui expriment la vérité des souffrances du locuteur.

Dans le «Au Lecteur » qui précède l'ensemble nommé Torrent des Sonets, Beaujeu revient sur le choix de ce nom. Il insiste, de nouveau, sur la « consonance » particulière de ses vers :

\begin{abstract}
Amy Lecteur, je donne plutost ce nom [Torrent des Sonets] à mes Sonets suivans, pour te donner courage de les lire, que pour autre chose, neantmoins je t'ay bien voulu advertir que si tu y prends bien garde, tu trouveras, non des vers frians et doux : mais rudes, et soubs la rudesse de leur consonance, de fort belles Histoires, et aussi esmerveillables que mal mises en œuvre. ${ }^{47}$
\end{abstract}

L'expression « la rudesse de leur consonance » fait naître l'idée d'une poésie peu agréable à l'oreille ou, en tout cas, dans laquelle la matière sonore vient heurter l'oreille du lecteur. Le locuteur invite celui-ci à découvrir, sous l'aspect peu engageant de cette poésie, un enseignement qui mérite que l'on s'accommode de la difficulté première. La promesse de «belles Histoires [...] esmerveillables » révèle un appel à une lecture attentive qui doit dépasser l'apparence pour entrer dans le secret d'un texte dont il s'agit de pénétrer les complexités. Il y a donc un gain à faire preuve de "rudesse », en ce que cette rudesse permet de dissimuler un sens à l'intérieur du poème tout en opérant un tri dans un public, entre les « ignorants » et les « savants ». Le lien entre l'écriture poétique, d'une part, et l'activité guerrière et ses aléas, d'autre part, est tout à fait revendiqué, car deux niveaux de lecture sont prévus par la périgraphie de ce recueil, niveaux qui

46. Beaujeu, Les Amours, f. 6 ro.

47. Beaujeu, Les Amours, f. 31 v $^{\circ}$. 
dressent à la fois un portrait du locuteur en tant qu'écrivain et une figure de lecteur.

L'insistance sur le terme "rude » fait écho à l'opposition entre le style bas et le style âpre telle qu'elle a été mise en scène par Ronsard lui-même dans l'évolution de sa manière entre les Odes et les Amours de 1552, d'un côté, et la Continuation et la Nouvelle Continuation ${ }^{48}$, de l'autre. Il entendait qualifier par là le passage d'une poésie érudite, saturée de références mythologiques complexes à une poésie faisant une place plus grande à la sensualité et à la nature qu'André Gendre ${ }^{49}$, à la suite de Ronsard, caractérise par le mot « doux ». Le poète en est, de fait, bien conscient, qui indique :

Il [Amour] suffit qu'on luy chante au vray ses passions

Sans enflure ni fard, d'un mignard et doux stile,

Coulant d'un petit bruit, comme une eau qui distile $\left[\ldots . . .^{50}\right.$

Est à nouveau développée ici une image fluviale ${ }^{51}$ pour rendre compte de la manière poétique : à l'opposition style " grave » / style " doux » se superpose l'opposition métaphorique « torrent » / « eau qui distile ». L'attention à la qualité sonore des vers est au cœur du discours ronsardien qui valorise, pour la lyrique amoureuse, un " petit bruit ». Beaujeu revendique donc une posture bien différente de celle du Ronsard des deux Continuations, en mettant en avant la « rudesse de [la] consonance de ses vers».

Loin de suivre le changement de vision poétique qui s'opère chez le chef de file de la Pléiade ${ }^{52}$, c'est à rebours que se construit la mise en scène

48. Ronsard, Euvres complètes (Paris : Société des Textes Français Modernes), vol. 1 à 5.

49. André Gendre, Ronsard, poète de la conquête amoureuse (Lausanne : Éd. de la Baconnière, 1970), 254-256. Ce critique rappelle également que la rupture entre les deux styles ne saurait être aussi nette et que les deux sont en fait concomitants. Tout juste peut-on voir des prédominances à telle ou telle époque.

50. Ronsard, Euvres complètes, vol. 7 (Paris : Société des Textes Français Modernes, 1959), « Élégie à son livre ", v. 178-180, 324.

51. Dans l'Antiquité, c'est Homère qui apparaît comme la mer dont sortent les fleuves représentant les grands auteurs. Sur ce point, voir Perrine Galland-Hallyn, Les Yeux de l'éloquence. Poétiques humanistes de l'évidence (Orléans : Paradigme, 1995), 174.

52. De même que pour le «torrent », la position de Ronsard vis-à-vis du couple « rude / doux » n'a cessé de fluctuer au cours du siècle, le mouvement le plus notable restant le passage du " rude » au " doux », de 
de soi que nous livre Beaujeu. L'élégie du feuillet $27 \mathrm{r}^{\circ}$ nous livre le récit de la formation poétique de Beaujeu, qui annonce avoir transformé sa manière d'écrire à cause d'une impossible communication avec la dame. Surtout, c'est un parcours inverse de celui décrit par Ronsard qui nous est dépeint, parcours lié aux expériences négatives du locuteur et non à une réflexion sur l'histoire littéraire et l'imitation des Anciens ${ }^{53}$ :

Aussi tost plein d'ardeur, et d'estranges envies,

Les Muses de mon cœur furent toujours suivies,

Sans cesse je chantois, et au gré de mon sort,

Le Cigne j'imitois, ignorant de ma mort,

Cuidant avec mes vers amollir la rudesse,

Et le brave desdain de ma jeune maistresse. ${ }^{54}$

Les débuts poétiques du locuteur nous sont narrés sous le jour le plus euphorique. Confiant dans son talent et mû par une passion que lui-même ne semble pas comprendre, le jeune poète se lance dans une pratique régulière de la poésie avec pour objectif clair de faire évoluer son amante. "L'ardeur » qui le pousse, ses « envies » et son application à suivre les Muses, disent assez tous les espoirs qu'il place dans la pratique poétique. La locution adverbiale " sans cesse » note à la fois la régularité et la durée de cette passion dévorante, tandis que l'image du « cœur " suivant les "Muses " semble renvoyer à un exercice constant de la science que les déesses lui accordent. Cependant, dès cette première strophe, des éléments dystopiques interviennent. Le verbe « cuider », d'une part, indique que les espoirs du jeune poète ne sont plus ceux

Pindare à Anacréon aux alentours de 1552-1553. Sur le style doux chez les auteurs latins et notamment Horace, voir Dominique Voisin, «Existe-t-il un genre de la douceur chez Horace ? Du vocabulaire de la douceur au genre de la douceur ", in La Douceur en littérature de l'Antiquité au XVII siècle, éd. Hélène Baby et Josiane Rieu (Paris : Classiques Garnier, 2012), 535-549 et, sur l'opposition et l'évolution du rude et du doux chez Ronsard, André Gendre « L'essence qualitative du "doux" chez Ronsard, Du Bellay et Baïf ", in Le "Doux » aux XVI et XVII siècles. Écriture, esthétique, politique, spiritualité, actes du colloque des 28 et 29 mars 2003, éd. Marie-Hélène Prat et Pierre Servet (Genève : Droz, 2004), 61-79.

53. De fait, dans l'" Élégie à son livre ", Ronsard (Euvres complètes, vol. VII, v. 178 et suivants, 324) nomme les auteurs qui doivent l'inspirer dans sa recherche d'une nouvelle manière et introduit une rupture stylistique entre l'âpreté tragique et la douceur de la parole amoureuse.

54. Beaujeu, Les Amours, f. 30 ro. 
du locuteur, la foi dans l'efficacité poétique n'est plus si forte. D’autre part, la mention du «Cigne», connoté positivement en tant qu'animal capable d'une production esthétique de grande valeur, est aussitôt modalisée par le second hémistiche du vers. De fait, le locuteur y déplore une erreur de jugement du jeune poète. Il convoque, dès lors, le topos du cygne produisant sa plus belle œuvre au moment de sa mort et semble dénigrer une poésie qui « amolli[ $\mathrm{t}]$ » : c'est en prenant conscience de ses souffrances qu'il parviendra au sommet de sa pratique poétique. Ces deux décrochages énonciatifs font apparaître une réflexion sur soi et préparent le lecteur à voir déçus les espoirs du jeune poète :

Mais las quand je congneu implacable mon mal,

Laissay de ma raison, j'imitay l'animal

Qui plein de desespoir seul tyran de sa vie,

A force de crier pert ame et fantasie,

Ou s'il voit que le temps ne soit pas assez prompt,

En cent mille façons son estomach il rompt,

Justement despité contre la mort cruelle $[\ldots] .^{55}$

Aux débuts idylliques succèdent le fantasme d'une auto-dévoration dans laquelle le poète devient fou-furieux. L'expression "laissay de ma raison » ne laisse aucun doute sur la perte de sens du locuteur. Celle-ci le pousse à quitter sa précédente manière poétique pour se tourner vers une esthétique de l'outrance, où la violence succède à l'abattement. La rupture de ton est marquée par la gradation, qui va de l'expression vigoureuse du malheur à travers les cris, qui n'ont ici plus rien de poétique, jusqu'à la destruction de soi. La «fantasie » perdue accentue la qualification des vers du poète ainsi changés puisque le lyrisme qui n'a plus accès à l'imagination se donne maintenant sous les aspects de la crudité. L'image de l'auto-dévoration peut se développer dans toute sa force. Le locuteur perd même son statut d'homme pour devenir une bête sauvage tâchant à tout prix d'échapper au « desespoir ». Ce n'est plus d'ailleurs la dame qui semble en cause, mais bien un « mal » non défini, auquel la «mort » doit apporter une fin. Celle-ci tardant, le locuteur lui attribue la continuation de ses souffrances et s'insurge contre elle. Ce passage se clôt par un énoncé à caractère gnomique dont la logique a quelque chose d'effrayant: 
Je treuve fort mauvais que nous n'avons mort qu'elle,

Il la faut mille fois au besoing demander,

Avant que l'on l'esmeuve à son arc desbander. ${ }^{56}$

Le nouveau rôle attribué par le poète à ses textes est clair : il s'agit véritablement d'invoquer la Mort ; mieux même, de parvenir à l'émouvoir. Le fantasme apocalyptique de l'existence de plusieurs Morts permettant une fin plus rapide entraîne une modification de la situation d'énonciation du texte, et non seulement de celui-ci, mais aussi de tous les autres poèmes puisque l'objectif va être de demander « mille fois » la mort en espérant l'obtenir. Le destinataire du texte, et avec lui celui de l'œuvre entière, a changé et la lamentation à laquelle va se livrer le poète emprunte sa " rudesse » non seulement aux souffrances subies et à la violence des expériences vécues, mais aussi à cette puissance destructrice qu'il s'agit de toucher et de pousser à réagir. Le locuteur se donne ici pour tâche ce qu'il considère lui-même comme hautement difficile à réaliser : l'oxymore de la mort émue. L'image macabre réactive celle du cygne poussant son plus beau chant à l'agonie. Le Torrent des Sonets s'annonce sous les auspices d'une mise en scène répétée et accentuée sans cesse des souffrances et des violences liées à l'amour, pour qu'enfin intervienne la libération du locuteur. Celle-ci ne peut cependant avoir lieu que dans une situation d'énonciation paradoxale où le poète avoue ne pas destiner son texte à un lectorat de ce monde, mais bien à des forces supérieures : on peut sans doute expliquer ainsi en partie l'étrangeté, la violence et la rugosité de ces poèmes.

\section{Conclusion}

Outre son statut problématique de soldat-écrivain, Beaujeu revendique des conceptions poétiques à rebours de l'évolution générale des pratiques littéraires de la fin du $\mathrm{XVI}^{\mathrm{e}}$ siècle. En ce sens, il semble vouloir occuper une position originale dans le paysage poétique des années 1580 , entre réactivation de certains termes utilisés dans les premières publications de la Pléiade et référence critique à l'évolution de la poétique ronsardienne. En 1589, soit quatre ans après la mort de son chef de file, il s'agit de prendre en main l'héritage de la Brigade, de se positionner par rapport à ses différents courants, tout en 
proposant sa propre manière. Le choix de Beaujeu de mettre en avant la rugosité de son style et la sincérité de ses œuvres relève d'un parti pris conservateur et rapidement anachronique. On comprend dès lors un peu mieux l'obscurité qui, aujourd'hui encore, l'entoure. Celle-ci n'était sans doute pas voulue au départ et l'absence de lectorat qu'a subie toute l'œuvre de Beaujeu semble être une conséquence imprévue de cette volonté de se démarquer fortement du nouveau goût naissant. Le poète du Torrent des Sonets a souhaité produire une poésie érudite et complexe à la manière des premières œuvres du Vendômois, suivant en cela le "goût rétrograde des humanistes ${ }^{57}$. Il se fait l'écho d'une affection du lectorat pour la poésie des années 1550, encore vue comme un âge d'or, qu'on lit cependant comme celle d'un temps révolu et qui n'entre plus dans les processus d'imitation des poètes de sa génération dont les nouveaux modèles sont désormais Desportes et Du Perron ${ }^{58}$. Beaujeu a donc été victime de l'écart, grandissant, entre la forte estime pour Ronsard, qui persiste jusqu’au début du $\mathrm{XVII}$ siècle, et la réalité des pratiques poétiques qui s'éloignent progressivement de ce point de référence.

57. Faisant, Mort et résurrection de la Pléiade, 41 et 49.

58. Il est d'ailleurs probable que Du Perron aurait prononcé sur Beaujeu un jugement semblable à celui que lui inspire Ronsard, Perroniana, cité par Faisant, Mort et résurrection de la Pléiade, 67-68 : « [...] Ronsard avoit de la force mais point de politesse [...] en ses amours il est quasi ridicule, et il y a quelquefois du galimatias ». Ce dernier terme ne peut manquer de nous faire penser aux "vers hors de rime et de raison » que Beaujeu mentionnait justement pour demander la bienveillance d'un public dont les goûts en matière de poésie amoureuse ont bien changé en l'espace de trente ans. 
